\title{
THIN FLEXIBLE AND STRETCHABLE TACTILE SENSOR BASED ON A DEFORMABLE MICROWAVE TRANSMISSION LINE
}

\author{
Matthew E. D'Asaro, Daniel B. Sheen, and Jeffrey H. Lang* \\ Massachusetts Institute of Technology, Cambridge, Massachusetts, USA
}

\section{ABSTRACT}

This paper presents a MEMS tactile pressure sensor based on distributed microwave sensing. Rather than using arrays of capacitive or resistive pressure sensors as is currently the state of the art, the sensor reported here determines the degree and location of applied pressure by measuring reflections on a thin embedded microwave transmission line. This approach allows for a rugged wide-area sensor that is easily and inexpensively fabricated and which requires only a single two-conductor connection to external electronics. The sensor is both flexible and stretchable, a rare combination of features among other tactile sensors.

\section{INTRODUCTION}

\section{Current state of the art in tactile sensors}

Over the past decade there have been numerous publications on tactile sensors and skins aimed at replicating the human sense of touch in applications such as robotics, healthcare, and prosthetics [1][2][3]. A variety of sensing approaches are used [1], with the dominant ones being piezoresistive [4] and capacitive [5][6]. The trend has been toward increasingly complex, thin, and sophisticated skins. While impressive resolutions, sensitivities, and degrees of integration have been achieved, mechanical durability and inexpensive manufacturing, properties essential for widespread use of tactile skins, have not advanced proportionately.

All current tactile sensing skins use arrays of discrete sensors. Forming $\mathrm{X}-\mathrm{Y}$ arrays of devices, which is the standard technique in microelectronics, has the advantages of density and performance. However, in the unique application of tactile skins, it has serious drawbacks as well. Arrays, by definition, require a minimum of $2 \sqrt{N}$ wires for $N$ sensors if each sensor requires only two wires and can be addressed by row and column. While not a serious problem in rigid electronics, in flexible and stretchable devices making the interconnections and reliably connecting rows and columns to external electronics present serious challenges. Furthermore, arrays are intrinsically fragile as damaging a single device can disable an entire row and/or column of sensors.

Other methods have been proposed to address the interconnect challenge, most notably [7], which demonstrates the use of flexible embedded silicon "nanomembranes" containing traditional silicon electronics that could perform decoding or even wireless data transfer functions. However, the need still exists for simple inexpensive sensing skins that do not require advanced electronics, which would be expensive except at a huge scale.

\section{Microwave sensing concept}

The sensing approach presented here addresses the arrayinterconnect challenge by eliminating the array. Conceptually, the technology works by applying a high frequency pulse to a transmission line with an impedance discontinuity due to a depression of the dielectric caused by applied pressure. This discontinuity causes a portion of the pulse to be reflected back to the source. Through time domain reflectometry (TDR), the roundtrip time-of-flight of the pulse and its amplitude can be used to determine the location and magnitude of the depression and hence the applied pressure; see Figure 1. Thus, the transmission line becomes a two-terminal distributed sensor that can be snaked over a two-dimensional area.

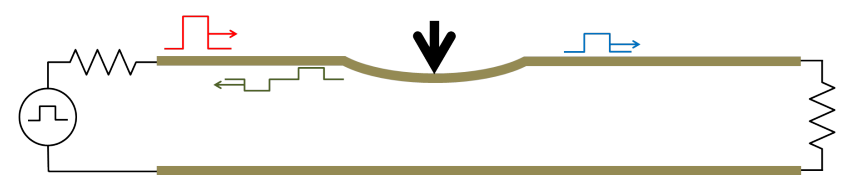

Figure 1: Concept for microwave transmission line sensing. In this conceptual drawing the red pulse from the generator is partially reflected off of the impedance discontinuity in the line caused by pressure (black arrow).

TDR is a standard technique for finding faults in cables, for example. While it works, TDR presents a few problems. First, if more than one discontinuity is present, the pulse can re-reflect between them causing "ghosts" in the response, which cannot be easily distinguished from genuine responses. Furthermore, a practical distributed pressure sensor will be exposed to continuous gradients of pressure, not discrete points, breaking the TDR analogy completely. For these reasons, the sensor is not excited here with a single pulse. Rather, its complex impedance is measured with a vector network analyzer (VNA) at 201 frequencies from about $30 \mathrm{MHz}$ to about $6 \mathrm{GHz}$. This data is then processed as described below to reconstruct the applied pressure.

\section{THEORY}

Reconstructing the deformation of the transmission line from measurements of its terminal impedance first requires understanding how the resulting line depressions perturb waves propagating along the line. To gain this understanding, the traditional Telegrapher's Equations are modified to be make the inductance per unit length $L(x)$ and the capacitance per unit length $C(x)$ functions of the position $x$ along the line. Thus,

$$
\begin{aligned}
& \frac{\partial V(x, t)}{\partial x}=-L(x) \frac{\partial I(x, t)}{\partial t} \\
& \frac{\partial I(x, t)}{\partial x}=-C(x) \frac{\partial V(x, t)}{\partial t}
\end{aligned}
$$

where $t$ is time, $V(x, t)$ is voltage and $I(x, t)$ is current.

To solve (1) and (2), several assumptions are made. First, assume the perturbation in line impedance is small, that is, the line is compressed less than about $10-20 \%$ of its thickness. Second, assume the line to be a lossless and properly terminated ideal parallel plate transmission line having length $l$ and plate gap spacing $g(x)$. With these assumptions, perturbation analysis yields

$V(x, t)=V^{*} e^{j \omega\left(t-\frac{x}{c}\right)}+\frac{V^{*}}{2 G_{0}} e^{j \omega\left(t-\frac{x}{c}\right)} \int_{x} \frac{d g\left(x^{\prime}\right)}{d x} e^{-2 j \omega \frac{x^{\prime}}{c}} d x^{\prime}$

where $G_{0}$ is the nominal conductor spacing with the line at rest, $g(x)$ is the perturbed conductor spacing as a function of position along the line, and $V^{*}$ is the amplitude of the wave launched at the terminals at $x=0$ with phase velocity $c$ and angular frequency $\omega$. In (3), the first term is a forward-traveling wave, and corresponds 
to the $0^{\text {th }}$-order term in the perturbation series. The second term is a backward-traveling wave that reflects off line depressions, and corresponds to the $1^{\text {st }}$-order term in the perturbation series. Thus, (1) and (2) are solved to first order in a perturbation parameter that scales $g(x) / G_{0}-1$. For $S_{11}(\omega)$ defined as the ratio of the backward-traveling wave to the forward-traveling wave at $x=0$, (3) can be rearranged to yield

$$
S_{11}(\omega)=\frac{1}{2 G_{0}} \int_{0}^{l} \frac{d g\left(x^{\prime}\right)}{d x} e^{-2 j \omega \frac{x^{\prime}}{c}} d x^{\prime}
$$

where $l$ is the length of the line and all other variables have been previously defined. $S_{11}(\omega)$ will be measured experimentally.

It is now desired to recover $g(x)$ from $S_{11}(\omega)$. To do so, observe that (4) is essentially a Fourier transform that can be inverted. Inversion is carried out numerically using a sampled measurement set. This results in

$$
\frac{d g}{d x}=\frac{4 N^{*} G_{0} f_{0}}{c} \operatorname{IFFT}\left[\mathrm{S}_{11}(\mathrm{f})\right] \frac{1}{\alpha}
$$

where $f=2 \pi \omega$ is circular frequency, $f_{0}$ is the frequency sample step size, and $c$ is the phase velocity in the transmission line which is slower than the speed of light by the velocity factor of the line. $N^{*}$ is the number of data points after the $S_{11}(f)$ measurement set has been made Hermitian so as to have a purely real inverse transform. This is accomplished by combining it with its complex conjugate and adding a DC reflection coefficient, which is assumed to be zero. The resulting measurement set has a length of $N^{*}=2 N+1$ where $N$ was the number of measured frequency points. The parameter $\alpha$ is defined as $G / Z \cdot d Z / d G$ where $Z$ is the impedance of the line and $G$ is the gap. For an ideal parallel plate transmission line $\alpha=1$ but due to the effects of fringing fields $\alpha$ is less than 1 for a real transmission line. Using the equations for the impedance of a real strip line transmission line given in [9] and the dimensions of the actual line, a value of $\alpha=0.6$ is derived.

Integrating (5) yields a reconstruction of the deformation of the transmission line gap as a function of position. Pressure is then calculated using the Young's modulus of the dielectric.

The distance between position data points $\Delta X=c /\left(2 N^{*} f_{0}\right)$ so the resolution of the sensor is limited by the shortest propagating wavelength. Thus, the resolution can be improved by either increasing the excitation frequency or decreasing the propagation speed. The frequency is limited to about $6 \mathrm{GHz}$ by loss and reflection due to manufacturing tolerances, but the wave speed can be decreased by increasing the dielectric constant of the PDMS dielectric. This is achieved, as in [8], by adding a high- $\kappa$ ceramic material, CCTO, to the PDMS.

\section{FABRICATION}

Much attention was paid during design to ensure that the sensors could be manufactured with simple molding techniques that can be easily scaled to arbitrarily large areas, as opposed to with lithographic techniques, which are generally limited by wafer sizes. Care was also taken to ensure insure that the completed sensors remained stretchable and flexible.

First, Polydimethylsiloxane (PDMS, Sylgard ${ }^{\circledR} 184$ ) is mixed with $10 \%$ linker and optionally CCTO powder in a centrifugal mixer (Kurabo Mazerustar KK-250S). Next a piece of stretchable silver cloth, Statex Shieldex ${ }^{\circledR}$ MedTex $^{\mathrm{TM}} \mathrm{P}-130$ is cut to be slightly larger than the finished line, soaked in pure PDMS and

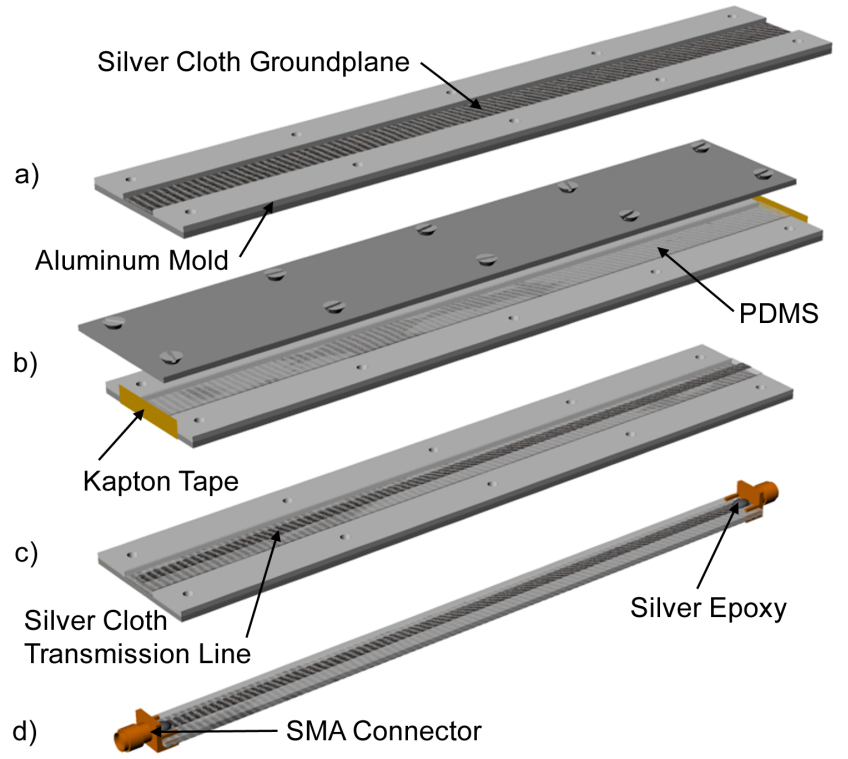

Figure 2: Fabrication diagram for transmission line pressure sensor. a) The silver cloth for the ground plane is soaked in PDMS, mounted in the mold and cured. b) The mold is filled with PDMS (optionally doped with CCTO), the cover is attached, and the PDMS is cured. c) The silver cloth transmission line soaked in PDMS and protected with Kapton ${ }^{\circledR}$ tape is installed and cured. d) The line is removed from the mold, the tape is removed, the excess silver cloth ground plane is trimmed and the SMA connectors are installed with conductive silver epoxy.

clamped in the mold. Before soaking, two small pieces of Kapton ${ }^{\circledR}$ tape are used to protect the back of this cloth from the PDMS where the SMA connectors will attach. The mold is then placed in a vacuum chamber until all air is removed. It is then cured in an oven at $120{ }^{\circ} \mathrm{C}$ for 20 minutes. With the mold thus prepared, it is filled with PDMS (optionally doped with CCTO powder) to form the dielectric. Again it is degassed; the cover is bolted onto it, and it is again cured. Next, another piece of silver cloth is protected with Kapton ${ }^{\circledR}$ and then precisely cut into a 4.1mm-wide strip, calculated to achieve a resting impedance of $50 \Omega$. After being soaked in pure PDMS, this strip is adhered to the top of the dielectric, tape side up. The lid is then replaced on the mold and it is cured one final time. Finally, the mold is disassembled, the excess silver cloth ground plane is cut off, and SMA connectors are attached to either end with MG Chemicals MG8331 conductive silver epoxy. See the fabrication diagram in Figure 2 and the finished sensor attached to a glove in Figure 3.

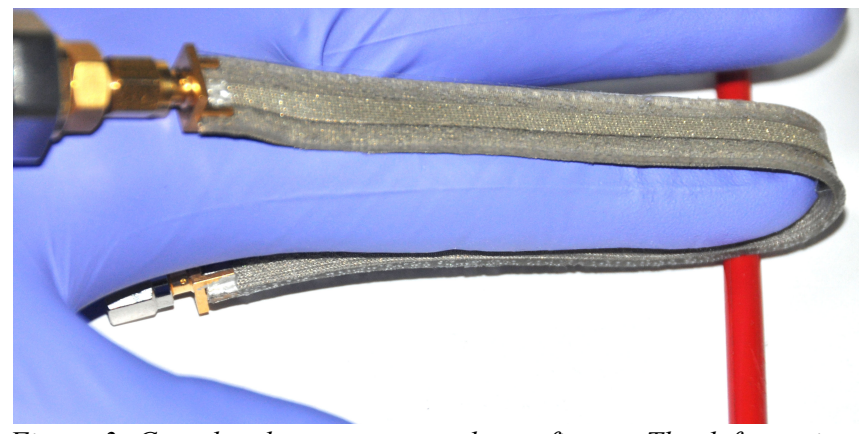

Figure 3: Completed sensor mounted on a finger. The deformation caused by pressing on the red plastic rod is easily detected. 


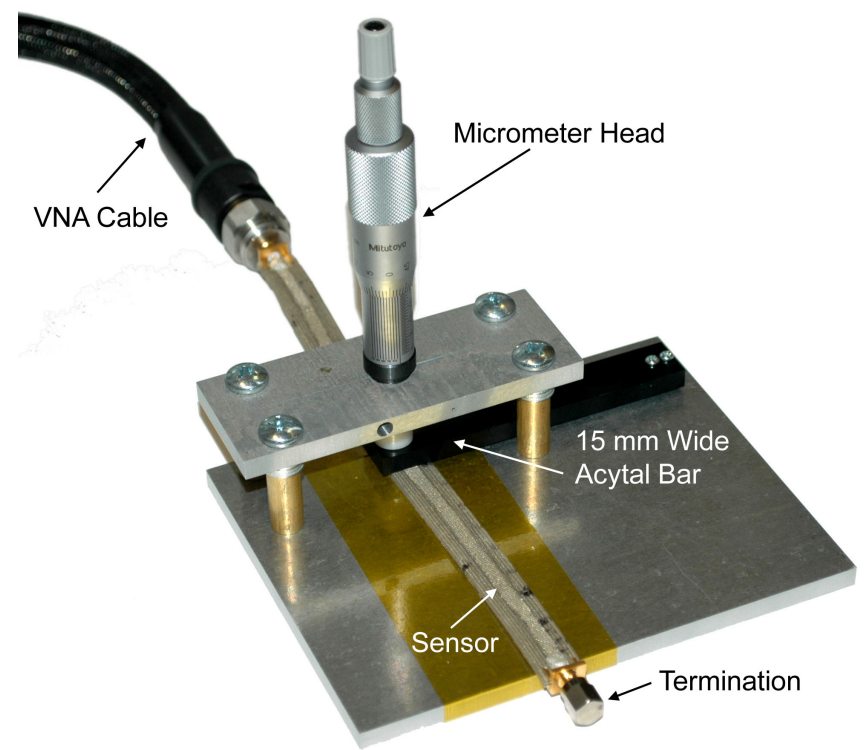

Figure 4: Device used for applying precise depression to the transmission line.

\section{Testing Procedure}

For testing, one end of the line end is connected to Port 1 of an HP $8410 \mathrm{C}$ vector network analyzer (VNA) with a flexible phase stable test port extension cable; the other end is terminated by a $50 \Omega$ microwave terminator. To apply a known depression to the line, the apparatus shown in Figure 4 is used. It consists of a micrometer head with a non-rotating spindle that presses a $15 \mathrm{~mm}$ wide piece of acrylic onto the line surface. Because the line is not shielded, care must be taken to avoid conductive or magnetic materials in close proximity with the sensor top. This is achieved by protecting the micrometer spindle with acetal.

The network analyzer is connected to a computer running a real-time version of the algorithm described above that allows the effects of depressing the line to be seen immediately. The software can also perform base-line subtraction on the collected data. This is necessary due to imperfections in line fabrication that result in permanent impedance discontinuities that would otherwise appear in the data. The baseline is subtracted after the line is inserted into the test apparatus and after the apparatus has been set to just begin depressing the line. This has the benefit of also subtracting off any effect due to the proximity of the spindle to the line.

To measure the position at which pressure was applied, the line was marked with a ruler and pressure was applied at the marked points. Note that zero position refers to the calibration plane of the vector network analyzer which is about $9 \mathrm{~mm}$ from the beginning of the active portion of the sensor.

\section{RESULTS AND DISCUSSION Position and Accuracy}

The sensor is very accurate in position, as position accuracy is affected only by the velocity factor of the line and the frequency accuracy of the VNA. Figure 5 shows the response of the line to depressions made at $40 \mathrm{~mm}, 100 \mathrm{~mm}$ and $160 \mathrm{~mm}$ beyond the calibration plane of the VNA. In each case the response is accurate to within the spacing of one data point $(7.3 \mathrm{~mm}$ for pure PDMS at $6 \mathrm{GHz}$ ). In this reconstruction a velocity factor of 0.585 was used, which matches the value of 0.584 found by directly measuring the propagation delay of the line with the VNA. Thus, the reconstruction appears to be quite accurate with regard to position.

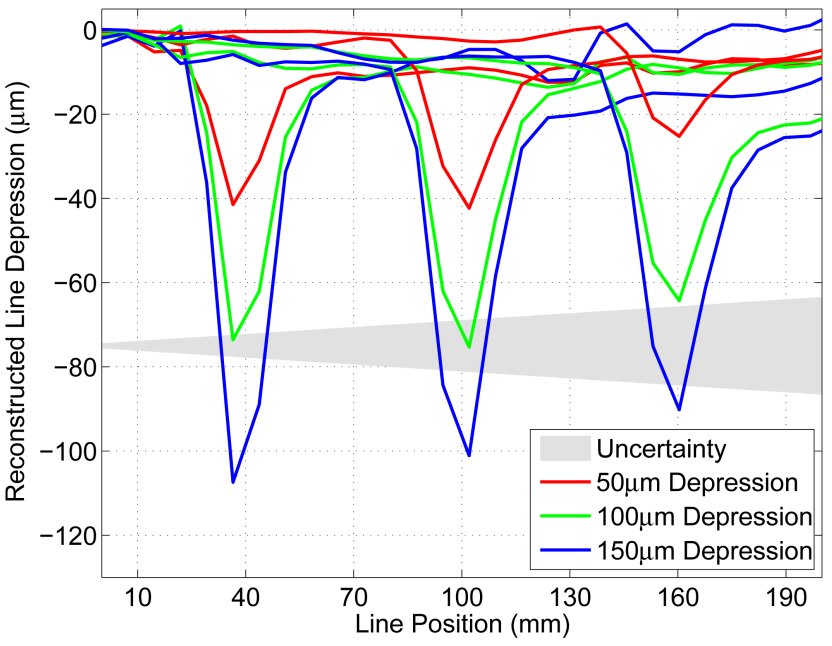

Figure 5: Plot showing depression response of transmission line sensor for three different pressure locations. The uncertainty trace represents 1 standard deviation.

\section{Depression Accuracy}

As seen in Figure 5, the depths of the depressions applied $40 \mathrm{~mm}$ from the start of the line are reconstructed to within $30 \%$ of their actual values. The discrepancy is believed to result from inaccuracy in the $\alpha$ parameter, which was calculated assuming a lossless transmission line. Indeed, experiments with a low-loss line made with copper foil conductors show agreement with theory at $40 \mathrm{~mm}$ to within $6 \%$. The response drops off more at positions farther away from the start of the line. This is primarily due to loss in the transmission line; from end to end it has a total resistance of $15 \Omega$ due to the resistance of the silver cloth. In future devices this will be addressed with improved reconstruction algorithms and/or more highly conductive stretchable materials.

A second effect seen in the data is that the depression settles to a value less than zero for positions along the line beyond the deformed region. This effect has been demonstrated through experiment and simulation to be due primarily to the resistance of the cloth decreasing locally where pressure is applied.

A third source of error is observed for narrowly deflected regions, narrower than a wavelength, for which the response is dramatically decreased. Experiments with simulated data that is otherwise free from error show that the response to depressions having a $10-\mathrm{mm}$ and $4-\mathrm{mm}$ width drops to $80 \%$ and $33 \%$ of normal, respectively. As a corollary, no conclusions can be drawn from features in a deformation plot that are smaller than about $15 \mathrm{~mm}$ due to the limited resolution.

Finally, note that the experimental uncertainty in Figure 5 increases with position along the line due to cumulative error in the integral used to reconstruct the depression. See Equation (4).

\section{Resolution and effect of added CCTO and Frequency}

Separate from the position accuracy discussed above, the sensor resolution must also be considered. The resolution is defined here as the distance between two discrete depressions at which they can no longer be distinguished from a single depression. To measure resolution, the sensor was depressed at two points with a constant force (from a weight) and these points were gradually brought together until the response from the sensor showed just one depression. At this point the distance between the points was measured with a ruler and recorded as the resolution.

The resolution (and the distance between individual position data points) of the sensor is limited by the size of the shortest 


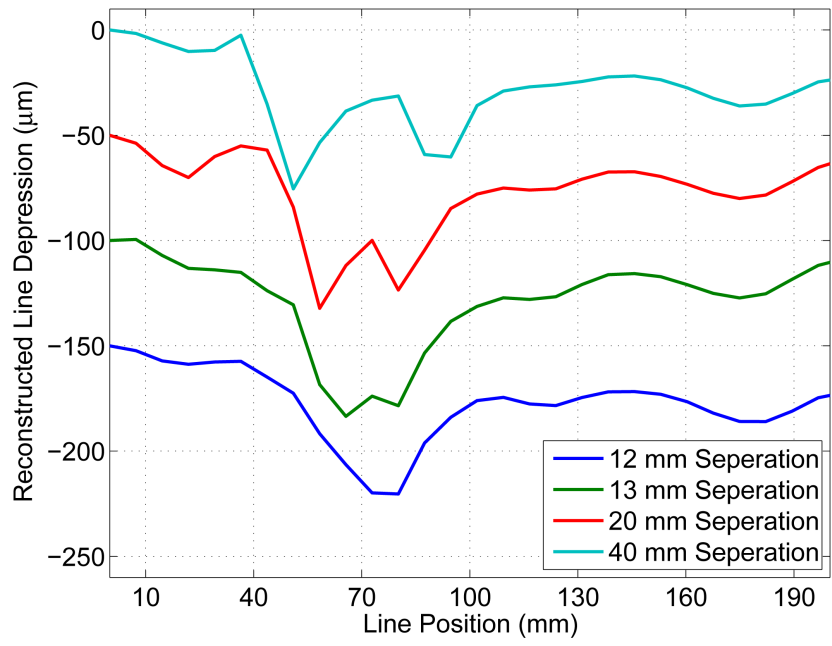

Figure 6: Plot showing resolution measurement. The measured resolution is $12 \mathrm{~mm}$ since that is the spacing at which the peaks converge. Curves have been staggered by $50 \mu \mathrm{m}$ for clarity.

wavelength used to excite it. Wavelength can be decreased by increasing the frequency, but the usefulness of increasing frequency is limited by the high frequency loss in the line. Wavelength can also be decreased by decreasing the velocity factor of the line which, per [8] can be achieved by adding high- $\kappa$ ceramic particles, such as CCTO to the PDMS. Table 1 lists the resolutions and velocity factors for sensors made with five different concentrations of CCTO. From the velocity factor, the wavelength at $6 \mathrm{GHz}$ was also computed. From this data two conclusions can be reached. First, the resolution of the sensor can indeed be increased by adding CCTO to the PDMS. The effect is modest though, at only about $20 \%$, a result consistent with that reported in [8]. Second, the minimum discernable resolution is consistently approximately half a wavelength and tracks with velocity factor demonstrating that the resolution limitations of the sensor are due to the wavelength of the propagating wave.

Table 1: Resolution as a function of CCTO concentration

\begin{tabular}{|l|l|l|l|}
\hline $\begin{array}{l}\text { CCTO } \\
\text { Concentration (\%) }\end{array}$ & $\begin{array}{l}\text { Resolution } \\
(\mathrm{mm})\end{array}$ & $\begin{array}{l}\text { Velocity } \\
\text { Factor }\end{array}$ & $\begin{array}{l}\text { Resolution / } \\
\text { Wavelength }\end{array}$ \\
\hline 0.00 & $12 \pm 1$ & $0.584 \pm 0.003$ & $0.411 \pm .036$ \\
\hline 6.31 & $12 \pm 1$ & $0.528 \pm 0.003$ & $0.455 \pm .041$ \\
\hline 10.2 & $12 \pm 1$ & $0.476 \pm 0.003$ & $0.505 \pm .046$ \\
\hline 15.4 & $11 \pm 1$ & $0.456 \pm 0.003$ & $0.482 \pm .047$ \\
\hline 20.3 & $9 \pm 1$ & $0.403 \pm 0.003$ & $0.447 \pm .053$ \\
\hline
\end{tabular}

\section{Determination of applied force from depression}

Due to the geometric complexity of deforming a line with an arbitrarily shaped object, the relationship between deformation and applied pressure is complex and beyond the scope of this paper. However, assuming the general shape of the line to be an elastic sheet, and that this geometry results in the PDMS behaving as a locally ideal spring, and further assuming that the Young's modulus of the sensor is equal to that of pure PDMS, $\sim 500 \mathrm{kPa}$, (the material becomes harder with added CCTO), the $10 \mathrm{kPa}$ pressure sensitivity of human skin [2] would result in a $37 \mu \mathrm{m}$ depression, which could easily be detected by the sensor. Thus, the device has a sensitivity approaching that of human skin.

\section{Other Considerations}

No attempt was made to measure the time response of the sensor because the slow VNA sweep rate dominates. However, it should be limited only by the mechanical response of the PDMS.

Due to aliasing, impedance measurement at sampled frequencies results in a limited maximum length of line that can be measured. However, for the $\sim 30 \mathrm{MHz}$ step size used here this length is nearly $3 \mathrm{~m}$. This effect is therefore not of practical concern.

At present, the major limiting factor to the practical application of this technology is the size and cost of the VNA required to measure the impedance data. However, portable VNAs exist; the Agilent FieldFox for example weighs only $3 \mathrm{~kg}$ and measures only $292 \times 188 \times 72 \mathrm{~mm}$. With the highly sophisticated RF electronics in cell phones it seems likely that wrist- or pocketsized custom electronics could be developed for this application.

\section{SUMMARY AND CONCLUSIONS}

While still in an early stage of development, the distributed microwave pressure sensor for tactile skins shows much promise. The resulting sensors are very simple to manufacture, flexible, stretchable, and quite durable. The sensors achieve a depression accuracy of $30 \%$ and a position accuracy of $7.3 \mathrm{~mm}$ for the pure PDMS sensor. The resolution and position accuracy can be increased by adding CCTO to the PDMS. The overall pressure sensitivity of the device approaches that of human skin.

\section{REFERENCES}

[1] C. Lucarotti et al., "Synthetic and Bio-Artificial Tactile Sensing: A Review", Sensors, vol. 13, no. 2, pp. 1435-1466, 2013.

[2] M. Hammock, et al., "25th Anniversary Article: The Evolution of Electronic Skin (E-Skin): A Brief History, Design Considerations, and Recent Progress", Advanced Materials, vol. 25, no. 42, pp. 5997-6038, 2013.

[3] T. Someya, "Building bionic skins", IEEE Spectrum, pp. 50-56, 2013.

[4] L. Wang et al., "Thin Flexible Pressure Sensor Array Based on Carbon Black/Silicone Rubber Nanocomposite", IEEE Sensors, vol. 9, no. 9, pp. 1130-1135, 2009

[5] L. Viry et al., "Flexible Three-Axial Force Sensor for Soft and Highly Sensitive Artificial Touch", Advanced Materials, vol. 26, no. 17, pp. 2659-2664, 2014.

[6] M. Cheng et al., "A Polymer-Based Capacitive Sensing Array for Normal and Shear Force Measurement", Sensors, vol 10, pp. 10211-10225, 2010.

[7] D. Kim et. al, "Epidermal Electronics", Science, vol. 333, no. 6044, pp. 838-843, 2011.

[8] L. K. Namitha and M. T. Sebastian, "Microwave dielectric properties of flexible silicone rubber - $\mathrm{Ba}\left(\mathrm{Zn}_{1 / 3} \mathrm{Ta}_{2 / 3}\right) \mathrm{O}_{3}$ composite substrates", Materials Research Bulletin, vol. 48, no. 11, pp. 4911-4916, 2013.

[9] D. Pozar, "Transmission Lines and Waveguides," in Microwave Engineering, 3rd ed. New York: Wiley, 2005, pp. 91-155.

\section{SUPPORT}

This work was supported by Award Number EEC-1028725 from the National Science Foundation. The content is solely the responsibility of the authors and does not necessarily represent the official views of the National Science Foundation.

\section{CONTACT}

*J.H. Lang, tel: +1-617-253-4687; LANG@MIT.EDU 